\title{
Acadêmicas de enfermagem frente as práticas educativas com gestantes: relato de experiência
}

\author{
Nursing students facing educational practices with pregnant women: experience report
}

Estudiantes de enfermería ante prácticas educativas con gestantes: relato de experiencia

Gabriela Aparecida Paz de Castro ${ }^{1 *}$, Adielly de Lima Souza ${ }^{1}$, Adriane Bonotto Salin.

\begin{abstract}
RESUMO
Objetivo: Relatar a experiência das atividades de educação em saúde realizadas com grupo de gestante em uma unidade básica de saúde em um município do estado de Rondônia, Brasil. Relato da experiência: Tratase de um relato de experiência, na visão de acadêmicas de enfermagem, sobre a educação em saúde para um grupo de gestantes, durante o estágio supervisionado I, em uma unidade de saúde da família (USF). A atividade foi supervisionada pela enfermeira preceptora e a enfermeira da USF. Foi realizado uma abordagem sobre cuidados com a gestante no pré-parto, orientações sobre o parto e cuidados como o binômio no pósparto, a fim de despertar interesse e favorecer a aprendizagem e interação das gestantes, optamos pela escolha de um vocabulário simples, utilização de estratégias pedagógicas, roda de conversa, e metodologias ativas. Considerações finais: Ficou evidente a importância das ações de enfermagem frente a essas atividades, pois através delas percebemos o quão necessárias são as atividades educativas, o contato com as gestantes levou a reflexão sobre as lacunas existentes na implementação da assistência de enfermagem no pré-natal, e o quão importante e valiosa ferramenta é a comunicação do enfermeiro com as gestantes usuárias do serviço.
\end{abstract}

Palavras-chave: Gestante, Estudantes de enfermagem, Educação em enfermagem, Saúde da mulher.

\begin{abstract}
Objective: To report the experience of health education activities carried out with a group of pregnant women in a basic health unit in a municipality in the state of Rondônia, Brazil. Experience report: This is an experience report, in the view of nursing students, about health education for a group of pregnant women, during the supervised internship I, in a family health unit (FHU). The activity was supervised by the governing nurse and the USF nurse. An approach was taken on care for pregnant women in the pre-delivery period, guidance on delivery and care such as the binomial in the postpartum period, in order to arouse interest and favor the learning and interaction of pregnant women. pedagogical strategies, conversation circle, and active methodologies. Final considerations: The importance of nursing actions in relation to these activities was evident, because through them we realized how necessary educational activities are, the contact with pregnant women led to reflection on the gaps in the implementation of nursing care in prenatal care, and how important and valuable the nurse's communication with the pregnant women who use the service is.
\end{abstract}

Key words: Pregnant woman, Nursing students, Nursing education, Women's health.

\section{RESUMEN}

Objetivo: Informar la experiencia de las actividades de educación en salud realizadas con un grupo de gestantes en una unidad básica de salud de una ciudad del estado de Rondônia, Brasil. Informe de experiencia: Se trata de un informe de experiencia, a juicio de los estudiantes de enfermería, sobre la educación en salud para un grupo de gestantes, durante el internado supervisado I, en una unidad de salud familiar (UFF). La actividad fue supervisada por la enfermera rectora y la enfermera de la USF. Se abordó el abordaje de la atención a la gestante en el período preparto, orientación sobre el parto y cuidados como el binomio en el posparto, con el fin de despertar el interés y favorecer el aprendizaje e interacción de la gestante. Estrategias pedagógicas, círculo de conversación. y metodologías activas. Consideraciones finales: Se

${ }^{1}$ Faculdade Interamericana de Porto Velho - UNIRON, Porto Velho - RO. *E-mail: gabrielanet9090@gmail.com 
evidenció la importancia de las acciones de enfermería en relación a estas actividades, pues a través de ellas nos dimos cuenta de lo necesarias que son las actividades educativas, el contacto con las gestantes llevó a la reflexión sobre las brechas en la implementación de los cuidados de enfermería en el prenatal, y lo importante y valiosa que es la comunicación de la enfermera con las embarazadas que utilizan el servicio.

Palabras clave: Embarazada, Estudiantes de enfermería, Educación en enfermería, Salud de la mujer.

\section{INTRODUÇÃO}

A fase do ciclo gestacional e puerperal é um momento especial na vida da mulher, uma experiência única, incomparável a qualquer outra vivência. No entanto pode ser permeado por diversas alterações físicas, emocionais e sociais. Gestar é um evento fisiológico, contudo existem múltiplos fatores que interferem na experiência da maternidade pela grávida, sendo eles o planejamento dessa gestação, as características socioeconômicas, a rede de apoio e os fatores que alteram a evolução dessa gestação pode influenciar de maneira positiva ou negativa (FALAVINA LP, et al., 2018).

Vasconcelos MIO, et al. (2016) discorre que ao oferecer uma adequada assistência no pré-natal é possível prevenir, diagnosticar e tratar os eventos adversos na gestação, objetivando o bem-estar da gestante e de seu parceiro, além de realizar orientações sobre possíveis adversidades específicas do parto e sobre determinados cuidados ao recém-nascido, ou seja, o acolhimento e a humanização da assistência perinatal ao binômio mãe-bebê, remete a mudança nos índices de mortalidade materna e neonatal.

Para Dias EG, et al. (2018) as gestantes se apresentam mais seguras com as ações de educação em saúde desenvolvidas durante o pré-natal, onde as mesmas têm suas dúvidas sanadas recebendo esclarecimentos pertinentes e imprescindíveis para evitar a quebra da analogia de naturalidade do ciclo gravídico-puerperal.

A mulher que recebe informações e orientações referentes à gestação, parto e puerpério no decorrer do pré-natal, enfrentará esse processo com maior autoconfiança, equilíbrio e prazer, pois o déficit de informação pode causar preocupações desnecessárias e perspectivas frustradas (VASCONCELOS ML, et al., 2016).

A gestação e o parto são encarados como acontecimentos naturais e fisiológicos que participam da vida sexual e reprodutiva da mulher sendo que, a gravidez tem que ser acompanhada de maneira favorável e não invasiva, viabilizando que a parturiente tenha propriedade diante do seu trabalho de parto de forma ativa (DA SILVA DF, et al., 2018).

A educação em saúde representa um instrumento de grande valia para a Unidade de Saúde da Família (USF), uma vez que, permite desenvolver ações de prevenção e promoção da saúde. A educação em saúde é uma prática que se desenvolve de modo para uma construção e evolução da sociedade, tornando-se assim, a educação um processo simplificado para o alcance da saúde, e abrangendo as mais variadas formas de dimensões de formação da comunidade (QUENTAL LLC, et al., (2017).

Cardoso FR, et al. (2019) menciona que é na execução da educação em saúde que, as mulheres tem a oportunidade de compartilhar experiências e vivências, assim como podem ser ouvintes de relatos de outras participantes, que contribuirão para solidificar informações sobre o ciclo gravídico puerperal, o autor discorre ainda que as atividades desenvolvidas em grupos para gestantes devem contar com ambientes apropriado onde elas possam dividir suas ideias e compartilhamentos de saberes em busca de soluções das mais variadas dúvidas.

Neste contexto, o estudo teve como objetivo relatar a experiência das atividades de educação em saúde realizadas com grupo de gestante em uma unidade básica de saúde em um município do estado de Rondônia.

\section{RELATO DA EXPERIÊNCIA}

A atividade aconteceu durante o Estágio Supervisionado I, em uma Unidade de Saúde da Família (USF) em um município do estado de Rondônia, Brasil. Durante a prática, as acadêmicas promoveram um momento de educação em saúde para um grupo de gestantes cadastradas no serviço e acompanhadas pela enfermeira 
durante o seu pré-natal. A atividade foi desenvolvida pelas acadêmicas e supervisionada pela enfermeira preceptora e a enfermeira da USF, a ação ocorreu em um único dia, no mês de novembro 2020. O Grupo contou com auxílio da equipe na divulgação e envio dos convites, por meio de mensagem, via grupo de WhatsApp, organizado e administrado pela Enfermeira da Unidade e os Agentes Comunitários de saúde (ACS) auxiliaram na divulgação para cada gestante das microáreas.

Inicialmente, foi definido a temática que seria abordada, o planejamento, a construção de materiais e as atividades que poderiam ser realizadas, para desenvolver a prática assistencial de educação em saúde. Com anuência e apoio da direção da unidade que forneceu um ofício, as alunas conseguiram doações de fraldas e produtos de higiene para presentear as gestantes participantes durante o encontro, que em sua maioria eram carentes. As acadêmicas providenciaram um lanche e organizaram e improvisaram um pequeno estúdio uma sala da unidade para realizar um mini book de fotos individuais das gestantes participantes. Para decoração cada acadêmica contribuiu com objetos para composição do cenário sendo eles: Puff, tapete, cortina e peças de roupa, sapatos e roupinhas do bebê para ser fotografado e no dia do evento se revezaram nas funções de fotógrafos, maquiadores, estilistas, na recepção e acolhimento das gestantes.

Os temas foram discutidos previamente com a enfermeira, levando em consideração as fragilidades, anseios e dúvidas percebidas durante as consultas de enfermagem e que precisavam ser reforçados. Para o preparo dos assuntos, foi empregado evidências científicas sobre cuidados com novo ciclo gravídicopuerperal, a fim de despertar interesse e favorecer a aprendizagem e interação das gestantes, optamos pela escolha de um vocabulário simples, e utilização de estratégias pedagógicas e metodologias ativas, como: roda de conversa e simulações realísticas dos cuidados com a gestante, puérpera e com recém-nascido. Como recursos didáticos utilizamos: bonecas, banheira, toalha, fralda, bola de pilates, álcool, tecidos, puff e balões para ornamentação do ambiente, e um aparelho celular para realizar as fotografias das gestantes.

Iniciou-se a atividade com uma roda de conversa, para estimular a participação das gestantes aplicamos uma dinâmica com mitos e verdades, com perguntas previamente elaboradas abordando: sinais e sintomas do trabalho de parto, orientações sobre puerpério, lóquios, higiene materna, aleitamento materno, cuidados de higiene como $\mathrm{RN}$ e com o coto umbilical, consultas de retorno no puerpério.

As gestantes participaram ativamente e todos tiveram oportunidade de ensinar e aprender, as gestantes relataram vivências prévias, todas possuíam saberes prévios, embora muitos destes eram advindos vivencias e conhecimentos acumulados por gerações, na oportunidade alguns mitos puderam ser trabalhados com prudência a fim de não afetar questões culturais da família, muitas dúvidas emergiram sobre os assuntos.

No decorrer as acadêmicas realizaram orientações sobre as temáticas, priorizando contemplar as dúvidas que surgiram e desmitificar alguns mitos, respeitando as questões culturais de cada paciente, abordaram sobre o cuidado e o preparo das mamas durante a gravidez e no puerpério, a fim de favorecer o início e a continuidade da amamentação. Abordaram sobre cuidado e curativo no coto umbilical, higiene materna e do $\mathrm{RN}$, vestimentas do bebê, características e duração dos lóquios, retorno as consultas puerperais, e demostraram técnicas não invasivas de alívio da dor, o uso da bola de pilates, esclarecimento sobre os sinais e sintomas do início do trabalho de parto, e exercícios que favorecem a dilatação, insinuação e expulsão do feto.

Durante todas as atividades foi utilizada linguagem de fácil compreensão e simulações realísticas, e a fim de verificar a compreensão e entendimento do que estava sendo explanado, as gestantes eram convidadas a realizar a simulação. Com auxílio de uma boneca e uma banheira simulamos a higienização corporal e intima, a troca de fralda, o curativo e cuidados com coto umbilical, uso da bola de pilates para favorecem a dilatação e auxiliar no alívio, a demonstração do posicionamento e a pega correta, como intercalar as mamas de forma que o bebê consiga receber o leite rico em gordura.

Ao final do encontro foi realizado sorteio de brindes, oferecido um lanche saudável e as que desejassem ser fotografadas eram encaminhas para o mini estúdio improvisado e previamente decoradas, onde acadêmicas auxiliavam na vestimenta e realizam a mini sessão de fotos. Todas as fotos foram entregues a enfermeira da unidade que compartilhou com as gestantes através do grupo de WhatsApp, este momento 
final foi regado muita satisfação, alegria e emoções, tanto por parte das acadêmicas quanto das gestantes que foram agraciadas com conhecimento, um mimo para compor o enxoval do seu bebê e a realização de fotos.

\section{DISCUSSÃO}

Carvalho SOC (2020) descreve que o estágio supervisionado em enfermagem é um momento indispensável para articular a teoria com a prática, visando o crescimento do aluno a partir de competências profissionais adquiridas que vão favorecer o desenvolvimento das competências necessárias ao trabalho. Proporciona ao acadêmico a visão profissional de forma ampla e concreta, oferecendo experiências de âmbito técnico-científico, com o desenvolvimento de funções com responsabilidade, ética, liderança, capacidade de comunicação e tomada de decisões.

Da Silva WB, et al. (2019) descrevem que a educação em saúde deve contribuir com a mudança no estilo de vida, auxiliando no conhecimento, e como resultado propiciar bem-estar físico e emocional, e para isso é indispensável que a linguagem empregada seja simples e de fácil entendimento, e ao mesmo tempo promova uma reflexão colocando as gestantes como protagonistas e detentoras do poder e da autonomia dos cuidados com sua saúde e do bebê.

Melo RHV, et al. (2016) menciona que a roda de conversa, é um diálogo que que estimula troca de conhecimento e vivências de práticas, promoção da saúde entre discentes, profissionais e comunidade. Através de dinâmicas interativas, buscam soluções e saídas para a problemática, isso além de estimular os discentes na busca da valorização e alcance de competências e habilidades fundamentais para o futuro exercício profissional, a construção dialógica e uma visão crítica, tolerante, flexível de si mesmo e do outro

Gonçalves MF, et al. (2017) descreve que o pré-natal é o momento oportuno para que as atividades de educação em saúde sejam praticadas, favorecendo, assim, a desmitificação de algumas informações e a obtenção de conhecimentos novas condutas relacionadas a saúde e modificação de hábitos, estas práticas educacionais ajudam a gestante enfrentar esse período com mais segurança e prazer, uma que a falta de conhecimento gera preocupações e frustrações. Discorrem ainda que estas práticas contribuem para a construção do vínculo com a equipe e ainda entre o binômio.

Segundo Vasconcelos ML, et al. (2019) o nascimento de um filho gera um mix de sentimentos, o que exige não só da mãe, mas de todos os que dela cuidam, que o reconheçam e valorizem, pois, esses sentimentos podem gerar conflitos causados pela insegurança, medo e dúvida diante da situação imposta, principalmente quando é o primeiro filho. Portanto, o pré-natal torna-se um espaço favorável para os profissionais orientarem, aconselharem, observarem e interagirem com as futuras mães para que todas as suas dúvidas sejam esclarecidas e esclarecidas a tempo. Ouvir as incertezas trazidas pelas novas experiências, ser orientado no processo de assistência pré-natal para expressar amor e cuidar do bebê contribui para a construção de um vínculo positivo entre mãe e filho.

A educação em saúde é uma ferramenta relevante na assistência ao ciclo gravídico-puerperal visto que, a gravidez refere-se a um período sensível e causador de grandes dúvidas e anseios para os genitores e seus familiares (FÉLIX HCR, et al., 2019).

Fagundes DQ e Oliveira AE (2016) discorrem que as ações educativas desenvolvidas durante o pré-natal realizadas por meio de métodos participativos garantem a troca de conhecimento prévio das mulheres entre o grupo e favorecem novos aprendizados, além de auxiliar na quebra de tabus e mitos.

Segundo Castro GVDZB (2018) a simulação enriquece práticas psicomotoras, potencializa os processos de aprendizagem, otimiza o pensamento crítico, desenvolve habilidades de raciocínio além disso, estimula o envolvido à participação ativa, interligando habilidades práticas e teóricas, o que facilita o processo de aprendizado e melhora de competências do participante da simulação realizada.

Andrade RD, et al. (2015) descreve que, é necessário prestar atenção holística à mulher no pré-natal, considerando a perspectiva geral da cultura social e do contexto familiar. O profissional deve estar atento e capaz de compreender $\mathrm{e}$ atender as reais necessidades de cada mulher para qualificar $\mathrm{o}$ atendimento 
prestado, discorre ainda sobre o Programa de Assistência Integral à Saúde da Mulher (PAISM), que surgiu em 1984 com a proposta de tratar a mulher como objeto de cuidado, devendo ser considerada e auxiliada em sua singularidade, não focando apenas os aspectos biológicos, mas também considerar outros aspectos.

Dadalto MZ, et al. (2020) descrevem que a fotografia não é apenas uma criação ela e capaz de revelar a essência, e reforçar a identidade da gestante e seu pertencimento num grupo, contribuindo para a saúde emocional e afetiva. A realização da sessão fotográfica estabeleceu como um momento importante na melhora da autoestima das gestantes e contribuiu no fortalecimento do vínculo entre os profissionais de saúde da UBS, fato comprovado pelas acadêmicas que podiam observar que muitas gestantes entravam na sala tímidas e após iniciarmos a sessão de fotos e com palavras de incentivo era notório a satisfação demostra e o empoderamento.

Assim, esta ação contribuiu de forma qualitativa na formação acadêmica, pois além de proporcionar um maior aprendizado sobre a temática, contribuiu na compreensão da importância do diálogo entre profissional e cliente, da escuta ativa e da capacidade de estimular hábitos saudáveis e o autocuidado de maneira correta e atual respeitando a individualidade, bem com proporcionou uma reflexão sobre as lacunas existentes na implementação da assistência de enfermagem no pré-natal, e o quão importante e valiosa ferramenta é a comunicação do enfermeiro com as gestantes usuárias do serviço. Anseia-se que a exposição do relato de experiência sobre ações educativas com gestantes instigue a discussão sobre metodologias de intervenção em saúde, a fim de que seus efeitos levem a melhorias na qualidade de vida do binômio e da família.

\section{REFERÊNCIAS}

1. ANDRADE RD, et al. Fatores relacionados à saúde da mulher no puerpério e repercussões na saúde da criança. Esc Anna Nery, Rio de Janeiro, 2015; 19: 181-186.

2. CASTRO GVDZB. O ensino mediado pela simulação realística: atendimento de intercorrências de saúde por professores da educação infantil. Dissertação (pós-graduação em enfermagem) -Centro De Ciências Biológicas E da Saúde. Universidade Federal De São Carlos, São Carlos, 2018.

3. CARDOSO RF, et al. Educação em saúde na assistência pré-natal: revisão de literatura. Revista Eletrônica Acervo Saúde, 2019; 2: 1-8.

4. CARVALHO SOC. Reflexões acerca da experiência do Estágio Supervisionado em Educação Infantil. Práticas Educativas, Memórias e Oralidades, 2020; 2: 1-12.

5. DADALTO MZ, et al. LUMIAR: A GESTAÇÃO EM FOCO. Anais da Jornada Científica e Cultural, $2020 ; 2: 167-171$.

6. DA SILVA WB, et al. Educação em saúde acerca da prevenção da violência obstétrica: relato de experiência. Revista Eletrônica Acervo Saúde, 2019; 14: e1163.

7. DA SILVA DF, et al. Conhecimento da gestante sobre as posições do parto. Revista de Enfermagem da FACIPLAC, 2018; $2: 1-9$.

8. DIAS EG, et al. Ações do enfermeiro no pré-natal e a importância atribuída pelas gestantes. Sustinere, 2018; 54: 5262.

9. FALAVINA LP, et al. Hospitalização durante a gravidez segundo financiamento do parto: um estudo de base populacional. Revista da Escola de Enfermagem da USP, 2018; 2: e6234.

10. FÉLIX HCR, et al. Sinais de alerta e de trabalho de parto: conhecimento entre gestantes. Revista Brasileira de Saúde Materno Infantil, 2018; 3: e3829.

11. FAGUNDES DQ, OLIVEIRA AE. Educação em saúde no pré-natal a partir do referencial teórico de Paulo Freire. Trab educ saúde, 2016; 225: 223-243.

12. GONCALVES MF, et al. Pré-natal: preparo para o parto na atenção primária à saúde no sul do Brasil. Rev Gaúcha Enferm, 2017; 3: e0063.

13. MELO RHV, et al. Roda de Conversa: uma Articulação Solidária entre Ensino, Serviço e Comunidade. Rev bras educ med, 2016; 303: 301-309.

14. QUENTAL LLC, et al. Práticas educativas com gestantes na atenção primária à saúde. Rev enferm UFPE online, 2017; 336: 335-341.

15. SUHRE PB, et al. Sistematização da assistência de enfermagem: percepções de gestantes acompanhadas em uma unidade básica de saúde. Revista Espaço Ciência \& Saúde, 2017; 22: 20-31.

16. VASCONCELOS MIO, et al. Intervenção educativa em saúde com grupo de gestantes: estudantes de enfermagem em ação extensionista no interior do Ceará. Expressa Extensão, 2016; 110: 108-118.

17. VASCONCELOS ML, et al. Cuidar de crianças menores de seis meses no domicílio: vivência de mães primíparas. Esc Anna Nery, 2019; 3: e20180175. 